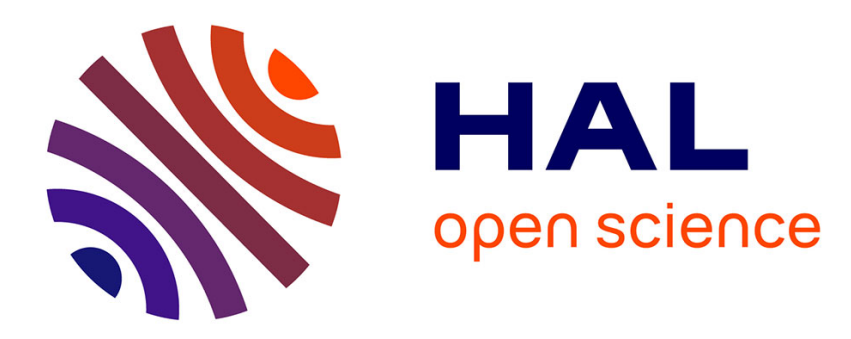

\title{
Nonequilibrium phenomena in lipid membrane phase transitions
}

\author{
P. Laggner
}

\section{To cite this version:}

P. Laggner. Nonequilibrium phenomena in lipid membrane phase transitions. Journal de Physique IV Proceedings, 1993, 03 (C1), pp.C1-259-C1-269. 10.1051/jp4:1993122 . jpa-00251565

\section{HAL Id: jpa-00251565 https://hal.science/jpa-00251565}

Submitted on 1 Jan 1993

HAL is a multi-disciplinary open access archive for the deposit and dissemination of scientific research documents, whether they are published or not. The documents may come from teaching and research institutions in France or abroad, or from public or private research centers.
L'archive ouverte pluridisciplinaire HAL, est destinée au dépôt et à la diffusion de documents scientifiques de niveau recherche, publiés ou non, émanant des établissements d'enseignement et de recherche français ou étrangers, des laboratoires publics ou privés. 


\title{
Nonequilibrium phenomena in lipid membrane phase transitions
}

\section{P. LAGGNER}

Institute of Biophysics and X-ray Structure Research, Austrian Academy of Sciences, Steyrergasse 17, 8010 Graz, Austria

\begin{abstract}
Time-resolved X-ray powder diffraction with synchrotron radiation has been applied to study the structural mechanisms of phase transitions in phospholipid model membrane systems at the millisecond time-scale. Transitions were triggered by single IR-pulses from an erbium glass laser. In those transitions where only the periodicity of the lamellar lattice changes, the transitions were found to be instantaneous, and interpreted as martensitic "Umklapp"-mechanisms. For transitions, which involve also a change in symmetry type, most notably the lamellar-to-rippled phase transition, short-lived ordered intermediates have been detected, which do not occur under near-equilibrium conditions. By prolonged incubation at the transition temperatures, it was found that defect structures are created which are conserved upon further changes in thermal conditions, indicating a memory phenomenon.
\end{abstract}

\section{INTRODUCTION}

Biological membranes can be viewed as two-dimensional complex fluid systems of lipids into which the functional, more or less rigid proteins are imbedded $/ 1 /$. The fluid mechanical properties are determined by the phospholipids which form the continuous structural matrix. The most common form is the phospholipid bilayer, however, increasing evidence points also to the significance of non-bilayer types, such as hexagonally packed tubes or cubic structures (Figure 1), which at least in isolated membrane lipids occur under conditions that can be considered as physiological (for reviews, see /2-5/). While any specific functional roles for this polymorphism are still rather hypothetical and poorly understood, it is wellestablished that the phase behavior of lipids can modulate the dynamic organization and activity of biomembranes (/6/). 


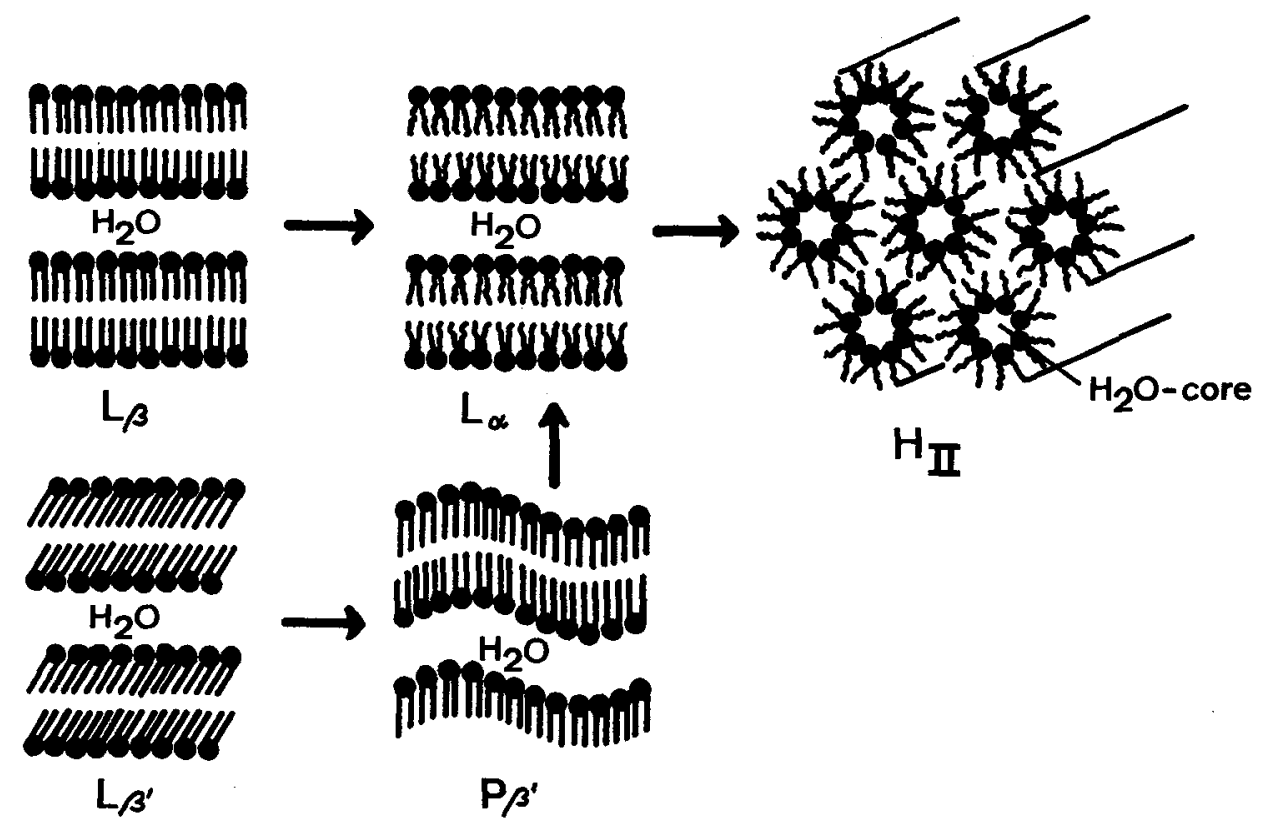

Figure 1: Structural scheme of some lipid polymorphic structures. Arrows indicate possible thermal transitions

While the equilibrium states of lipids are both structurally and thermodynamically well explored, very little is known about the processes of their interconversions, whether they are characterized by an intermediate loss in longrange order, or whether there exist specific topological pathways with preservation of connectivity. Such questions were so far beyond the reach of structural investigation through $\mathrm{X}$-ray diffraction techniques, simply because the necessary time for data acquisition, typically minutes to hours, was by orders of magnitude longer than the intrinsic transit times. Only recently, through the advent of synchrotron radiation sources with their abundant $X$-ray flux it has become possible to attempt real-time monitoring of structural phase transition processes by cinematographic diffraction /7-10/.

\section{SOME REMARKS ON METHODS}

Early experiments by the author $/ 7 /$ and other groups have shown that powerful synchrotron radiation would make it possible to reach time-resolutions of microseconds or even below, in studies of lipid phase transitions. The high $X-r a y$ flux of synchrotron radiation sources (for reviews, see $/ 11,12 /$ ) is only one of the important prereqisites to perform fast diffraction experiments on phase transitions. The other two are:

- rapid trigger methods to synchronize the transitions

- fast and efficient detector systems.

These are as important as the radiation source, and future progress will be strongly influenced by the development in these areas. In the following, some important details of these points will be considered. 


\section{T-jumps by IR-Laser}

Convective external heating, e.g. with a heat-gun or by switching between cold and hot thermostat fluids, is unsuitable for subsecond experiments, because any of those methods is limited by the low heat conductivities of the sample container and water as the solvent. The classical temperature-jump technique by electric discharge has other shortcomings, connected to the requirements of relatively high salt concentrations which may alter the phase behavior of the lipids a priori and thus render them unsuitable for these purposes. Of the two alternatives for radiative heating, IR-laser and microwaves, we have chosen to use IR-laser and obtained heating pulses in the order of $10^{\circ} \mathrm{C} / \mathrm{millisecond}$. Microwaves have been employed by Caffrey $/ 13 /$, but the heating rates were only in the range of $20-30^{\circ} \mathrm{C} /$ second.

The laser used in our work is an erbium glass-laser designed and built by Gert Rapp of the European Molecular Biology Laboratory /14/. It can be triggered to produce a 1 millisecond pulse (FWHM) with an emission wavelength of $1.54 \mu \mathrm{m}$, and an integral pulse energy of 1-2 Joules. This power can be applied over a sample area of approximately $2 \times 6 \mathrm{~mm}^{2}$. The effect of heating is produced mainly by IR-absorption in water, for which the absorption coefficient at this wavelength is $6.5 \mathrm{~cm}^{-1}$. By placing a gold-mirror underneath the exit face of the sample capillary of $1 \mathrm{~mm}$ diameter, and thus reflecting the transmitted laser beam, a T-jump of approx. $10^{\circ} \mathrm{C}$ with a maximum $\mathrm{T}$-difference of $2^{\circ} \mathrm{C}$ between the extreme positions in the sample can be produced. $\mathrm{A}$ detailed description is given in /15/. A schematic view of the experimental arrangement is shown in Figure 2.
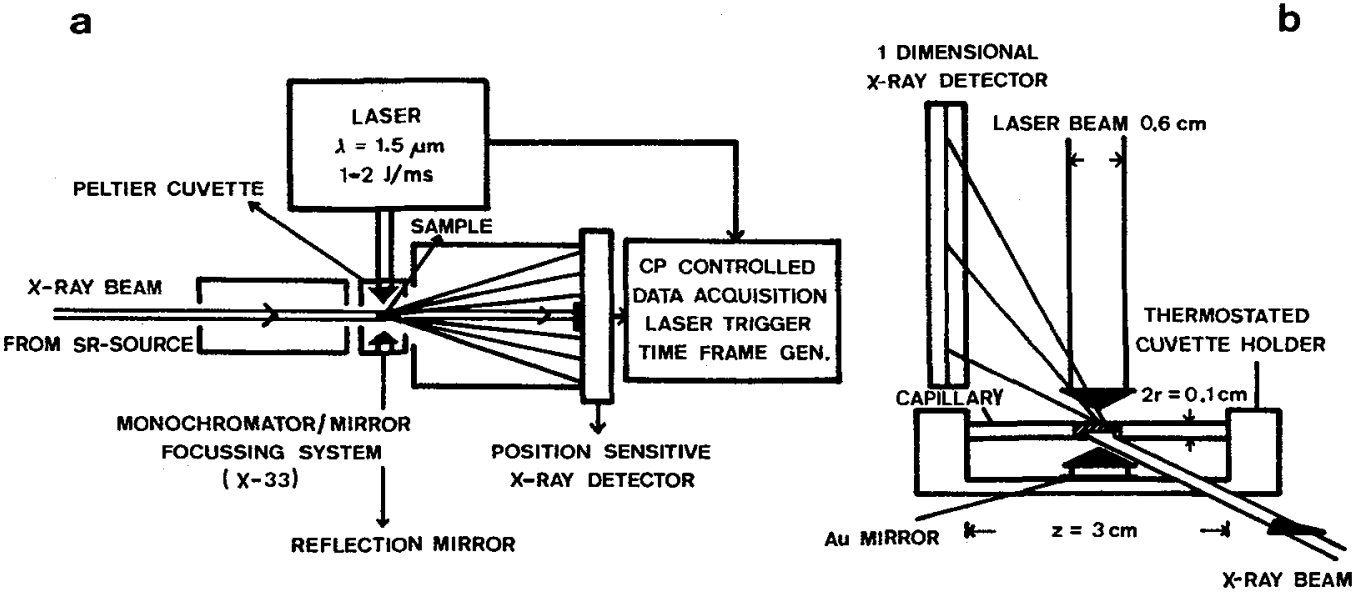

Figure 2:(a) Scheme of the IR-laser T-jump with time-resolved X-ray diffraction. (b) Position of the sample capillary in the $X$-ray and IR-beams, respectively (from /15/ with permission. Copyright by Elsevier Sci.Publ. B.V.) 
Passive heat dissipation within the observed sample volume is negligible for up to several seconds. Thus the relaxation behavior of a lipid sample can be recorded with ms-time-resolution for the first second or so after rapid and synchronous heating through a thermal transition, for which the calorimetric half-widths are typically in the order of less than $1^{\circ} \mathrm{C}$. A further improvement of the time-resolution can be expected from the development of a shorter laser pulse length and faster detectors.

It is important to note the basic difference between this approach and the classical T-jump method designed for the study of chemical reaction kinetics. The above experiments imply large jump amplitudes to cross the transition range rapidly, i.e. the system is driven far from equilibrium and consequently the thermodynamic driving force is high and, as seen from the comparison to experiments close to equilibrium (see below the case of the $L_{\beta} P_{\beta}$-transition), the response may become nonlinear. The classical chemical kinetics approach, on the other hand, attempts to minimize the jump amplitudes to determine the equilibrium reaction rates at a given temperature.

Although IR-absorption at present is the fastest method to induce phase transitions, this type of experiment has the principal shortcoming that it can only be applied in one direction, from the low to the high temperature phase. This problem can be overcome by pressure jump experiments, however, these are presently only in their infancy. Obviously both, $T-$ and $p$-jumps are thermodynamically related (as expressed through the Clausius-Clapeyron equation), and, experimentally the one is always associated by the other - in a counteractive fashion. T-jumps are limited by the phonon propagation, if the transition is associated by a volume discontinuity, and $p$-jumps necessarily involve a $T$-jump which reduces the effective volume work. Thus, the detailed quantitative assessment of such jump-experiments is by no means straightforward.

\section{SWAX: Simultaneous Small- and Wide-Angle Detection}

The structural changes of lipids during phase transitions are manifested in two distinctly different distance ranges. Changes in the long periods, repeat distances between the bimolecular layers, are typically in the range between 50 and $100 \AA$ (for cubic lattices even higher); the details of lateral hydrocarbon chain packing, on the other hand, are normally in the range of some 3-5 $\AA$. Therefore, with the reciprocal relationship between real-space distances and scattering angles, both small- and wide-angle diffraction patterns are highly relevant for a structural analysis. This, first of all, requires a good optical resolution of the camera at small angles ( $\leq 10$ mrad, for the typical wavelength of $1.5 \mathrm{~A})$, and at the same time well-resolved measurements between 15 and $30^{\circ}$. A scheme is shown in Figure 3.

No single electronic detector system was so far capable of satisfying both requirements - either the resolution is poor in the small-angle region, or it does not reach the wide angles. We have recently developed a camera employing two separate 
position sensitive detectors $/ 16 /$, which overcomes these difficulties, and is now available commercially (MBraun-Graz Optical Systems, Graz, Austria). A basically similar camera has been installed at the Hamburg synchrotron (EMBL station X-33 at HASYLAB; Rapp and Koch /17/).

wide angle

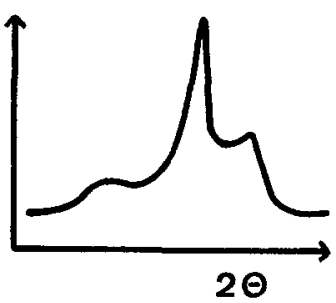

$2 \theta$

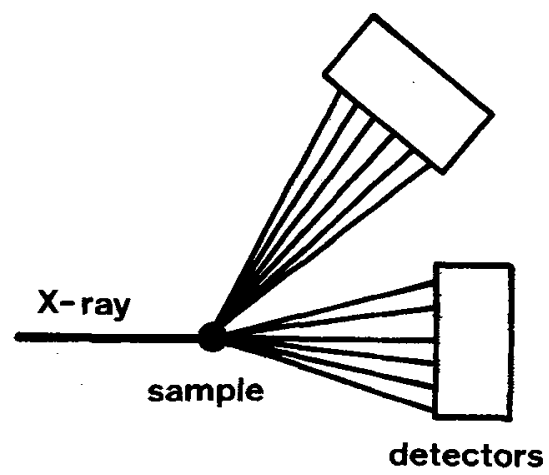

detectors

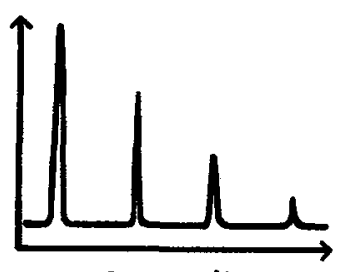

$s=2 \sin \theta / \lambda$

small angle chain packing
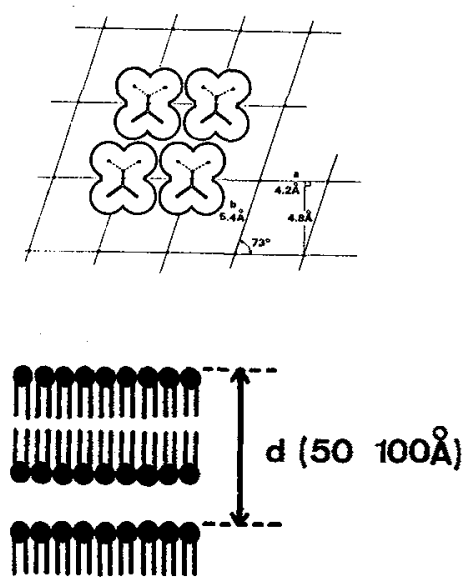

long periods

Figure 3: The two regions of interest in X-ray diffraction from membranes.

A further requirement on detector systems comes from the fact that in certain cases of cubic phases large, monocrystalline domains are formed, which consequently lead to streaks or spots rather than to powder diffraction circles. A linear redout in one dimension is therefore not sufficient to define the patterns. This means, that 2dimensional time-resolved detector systems have to be employed. Presently such systems are confined to a time-resolution in the order of seconds, and an improvement is hindered by the problems involved with the enormous data flux needed to handle 2-D patterns cinematographically.

\section{RESULTS FROM T-JUMPS}

In the discussion of structural phase transitions we have found it convenient to introduce an operational classification based on the geometric nature of the longrange rearrangements involved. Thus we use the term "homologous" for transitions between affine lattices, i.e. where only the lattice parameters change, while the symmetry type remains unchanged (e.g. lamellar-lamellar). "Heterologous" transitions, on the other hand, are those for which the symmetry type changes (e.g. lamellarhexagonal). Despite its simplicity and lack of theoretical foundation, it will be seen that this system allows for certain predictions regarding the transition mechanism. 


\section{Homologous Transitions : The Concept of Martensitic "Umklapp"-Transformations.}

A typical result of a T-ujump experiment for a transition between two lamellar lattices, lamellar-gel to lamellar liquid-crystalline $\left(L_{q}-L_{\beta}\right)$ of an ethanolamine phospholipid (1-stearoyl-2-oleoyl-sn-glycero-3-phosphoethanolamine, SOPE) in water is shown in Figure 4.

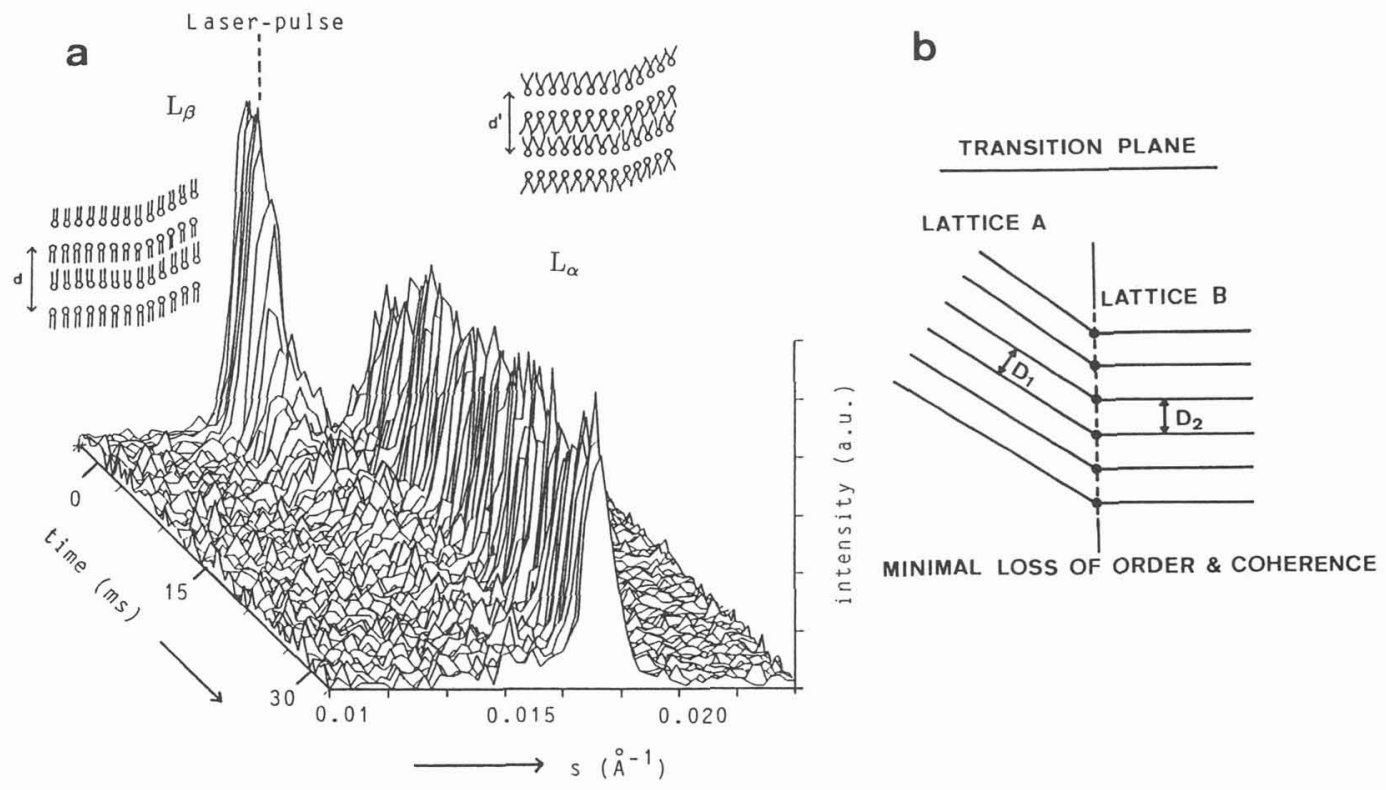

Figure 4:(a) time-development of the first-order lamellar repeat peak of the powder pattern from the SOPE/water system in a T-jump from $25^{\circ} \mathrm{C}$ to $32^{\circ} \mathrm{C}\left(\mathrm{L}_{\beta}-\mathrm{L}_{a}\right.$ transition), with a nominal resolution of $250 \mu \mathrm{s}$ per detection frame. The physically relevant resolution is given by the duration of the laser pulse of 1-2 ms. (b): scheme of a martensitic "Umklapp"-transition; the transition plane moves rapidly through the system.

At the time-scale of the experiment, this transition is immediate. The disappearance of the parent and the appearance of the nascent diffraction pattern coincide in time, and no intermediate product, ordered or disordered, can be detected. This leads to the conclusion that the transition is, at least at this time-scale and with respect to the long-range structural characteristics, a one-step, two-state process. From simultaneous wide-angle measurements it would appear that the chain-melting process fractionally precedes the long-range effects (Rapp and Rappoit, personal communication); this result, however, will have to be verified by further experiments.

In an attempt to rationalize these findings, in particular the notion of coexistence of the two lamellar structures with different repeat distances without intermediate disorder, we have postulated /18/ that the transition occurs by cooperative folding about a disclination plane, as indicated in Figure $4 \mathrm{~b}$, which 
propagates rapidly through the system. The ratio of the repeat distances is simply related to the cosine of the disclination angle. This mechanism accounts for the conservation in lattice order and for a minimum in defect formation. Transitions of this type are known to metallurgists as martensitic "Umklapp"-transitions $/ 19,20 /$.

\section{Heterologous Transitions: Structural Intermediates}

In these transitions, the lattice dimensions and the symmetry types are changed. The best studied cases are the lamellar to inverted-hexagonal $\left(\mathrm{L}_{\mathbb{n}}-\mathrm{H}_{\mathrm{II}}\right)$ and the lamellar-gel to ripple-phase $\left(L_{\beta}-P_{\beta^{\prime}}\right)$ transitions, respectively. In both cases, the temperature jumps have shown the existence of short-lived intermediates

The La-HIr Transition.

Figure 5 shows the time-resolved small-angle diffractogram in the region of the first- and second-order Bragg reflections together with the time-course of the changes in the peak positions.

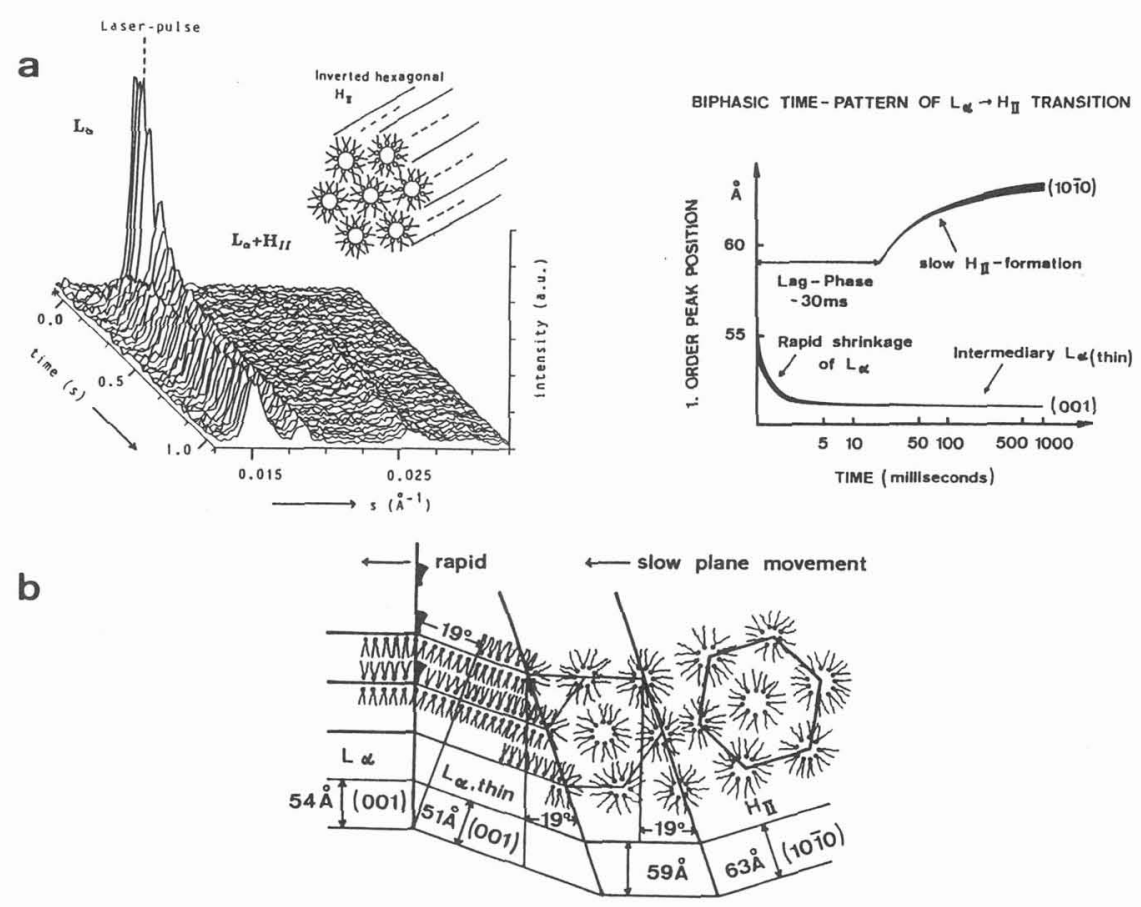

Figure 5: (a) Time-resolved small-angle diffractogram of the SOPE/water system, and time-dependence of the peak-positions. (b) Structural model for the $\mathrm{L}_{\mathrm{a}}-\mathrm{H}_{\mathrm{II}}$ transition mechanism.

Evidently there are two steps to be distinguished: a first step, occurring instantaneously, in which a thinner lamellar lattice is formed, and a second step starting after a lag-phase of some $20 \mathrm{~ms}$ in which the hexagonal lattice, initially distorted and in coexistence with the thin lamellar intermediate, is formed slowly with 
a completion time in the order of seconds. A scheme of the structural interpretation is also shown in Figure 5b. The first, rapid step is discussed again in terms of a martensitic Umklapp-process. This leads to the necessary close approach of adjacent headgroup regions, and must be associated with the rapid loss of some of the interlamellar water, or alternatively, with an increase in molecular area at the lipidwater interface. This step is also visible in slow temperature scan experiments, and it is, therefore, likely that this thin lamellar intermediate is thermodynamically stable. The second step is a relatively slow nucleation and growth process, characterized by the conservation of overall regularity as indicated by the low amount of diffuse small-angle scattering and the relative sharpness of the peaks.

\section{The $L_{\beta^{\prime}}-P_{\beta^{\prime}}$ Transition (Pretransition)}

In saturated diacyl-phosphatidylcholine lipids, there exists a stable ripple-phase $\left(P_{\beta^{\prime}}\right)$ between the lamellar gel $\left(L_{\beta^{\prime}}\right)$ and the lamellar liquid crystalline phase ( $L_{\alpha}$ ) /21/. Our time-resolved small-angle diffraction experiments /22/ have shown the following sequence of events (Figure 6a).

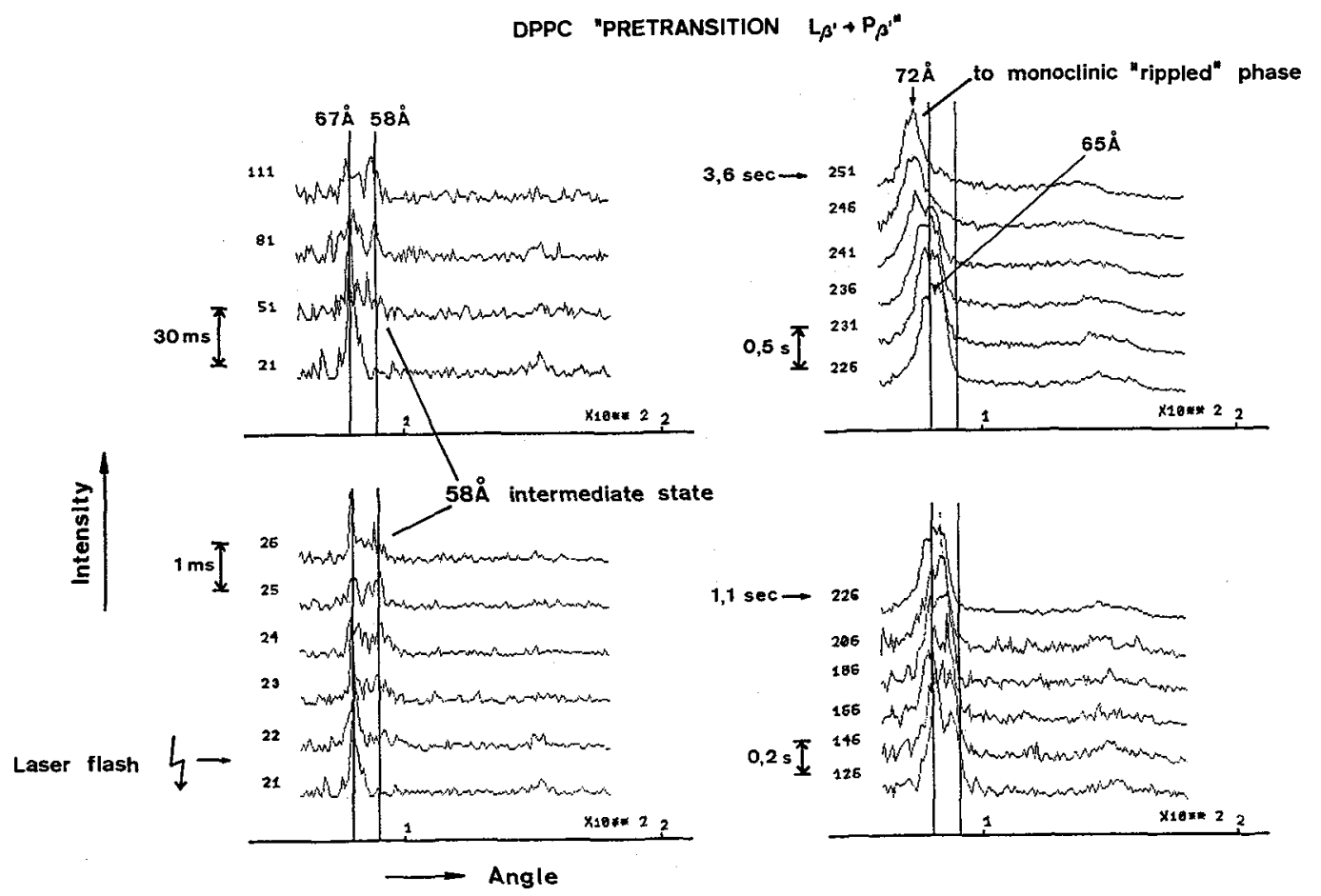

Figure 6a: Time-resolved small-angle diffractogram of the pretransition of DPPC/water (1,2-dipalmitoyl-sn-glycero-3-phosphocholine) after a laser-induced T-jump. 


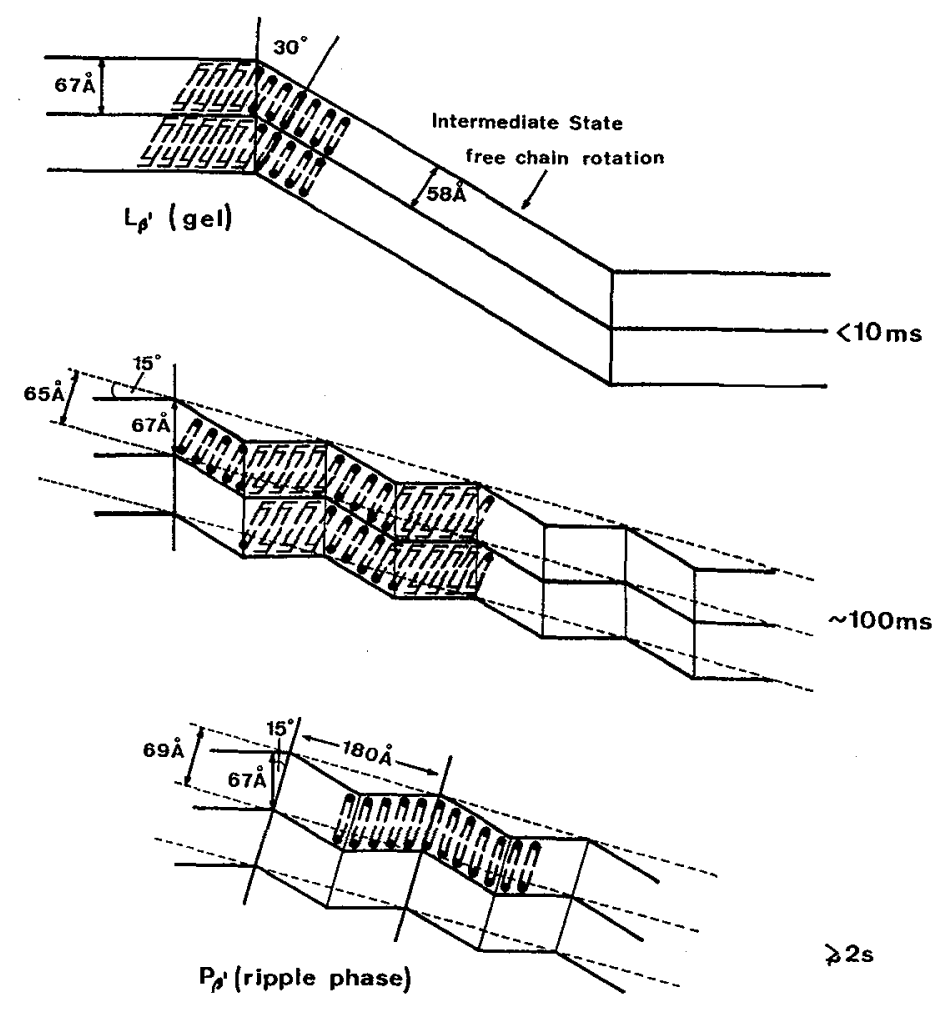

Figure 6b: Structural model of the mechanism for the DPPC-pretransition under Tjump conditions.

In a first step, occurring instantaneously at the experimental time scale, a lamellar lattice is formed with a smaller repeat distance, which coexists for various lengths of time (depending on salt conditions and the chain length of the lipids), with the parent lattice. These two lattices, as manifested by their distinct Bragg reflections at 64 and $58 \AA$, respectively, merge slowly over a time of several seconds to give way to the final lattice with a relatively broad first-order peak at about 72 \&. A tentative mechanistic model for this process is shown in Figure $6 b$. There remain several important questions, such as the time course of the chainpacking transition and the fate of the interbilayer water during the existence of the intermediate, thin lattice, which will have to be solved by future investigations. However, it is worth noting that this is the first verification of a dissipative intermediate structure in phase transitions of lipids, since this intermediate cannot be detected under equilibrium conditions. Thus, structural transitions can follow different pathways depending on the conditions and it is, therefore, important in the discussion of transition mechanisms to consider the power - and not just the energy - involved in triggering the transition. It is also tempting to speculate that such intermediate structures could play a biological role in signal reception and noisefiltering since they provide a mechanism for discerning high power signals from random noise. 


\section{Memory Effect}

Under isothermal conditions, i.e. upon incubation of a series of identical samples for extended periods (weeks) at different temperatures across the transition range, and small-angle scattering at exactly these temperatures, a completely different picture is obtained for the pretransition (Figure 7).

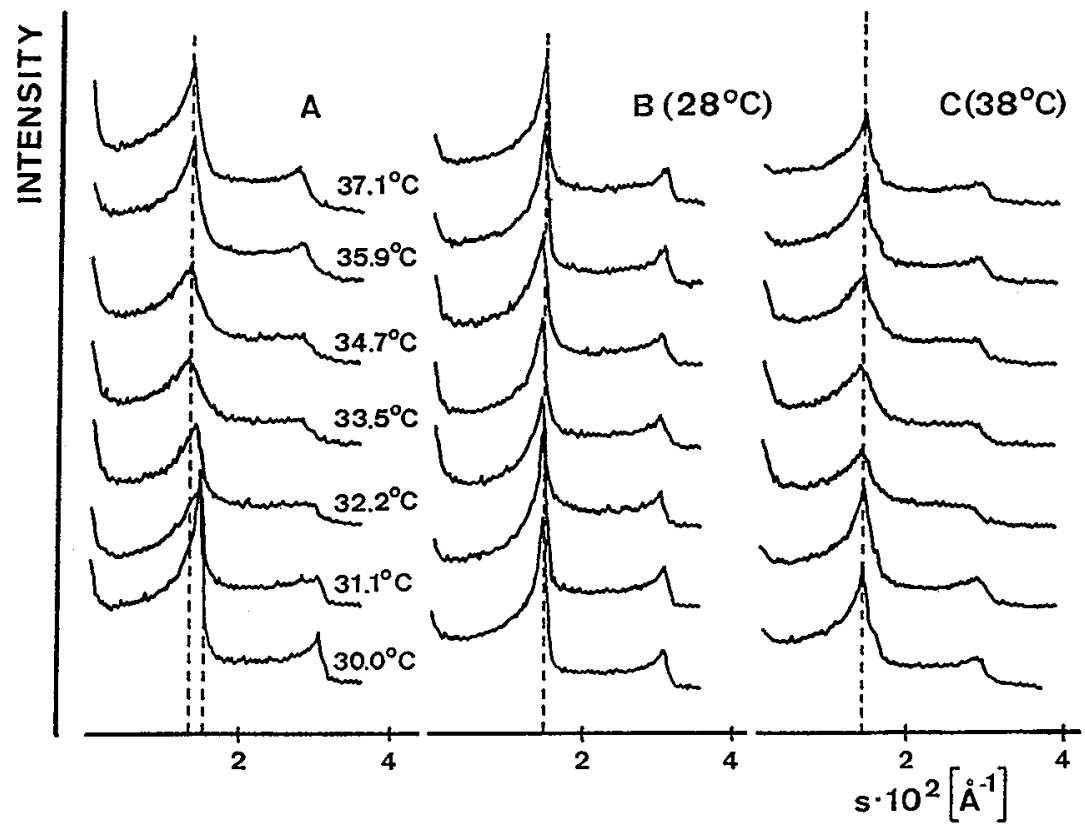

Figure 7: Small-angle-diffraction patterns of DPPC in excess water around the pretransition temperature. A: samples incubated for 2 weeks at the temperatures indicated; $B$ : samples from $A$, after cooling to $28^{\circ} \mathrm{C}$ and 2 days incubation; $\mathrm{C}$ : samples from $\mathrm{B}$, heated to $38^{\circ} \mathrm{C}$.

The diffraction patterns in the transition temperature range are considerably broader indicating loss of order. This can be considered as due to an accumulation of defects which appear to be very well-conserved as demonstrated by the following temperature protocol. Upon cooling of all samples to temperatures below the transition, i.e. to the $L_{\beta}$-phase,the Bragg peaks appear indistinguishably sharp for all samples. If these samples are then heated to well above the pretransition, into the domain of the ripple-phase, those samples which originally had been kept at the transition temperature, showed considerably broader peaks than the others, which had only in passing experienced the transition. Thus the samples keep a memory of the accumulation of defects they had acquired in the transition. A simple analogy of such a behavior would be a crumpled sheet of linen that can be straightened, but will arimnla anain at tho nroformod foldinn linac 


\section{ACKNOWLEDGEMENT}

This work has been supported by the österreichischer Fonds zur Förderung der Wissenschaftlichen Forschung under grant no. S4614. Many stimulating discussions with Drs. A.Bóta, M.Kriechbaum and G.Rapp are gratefully acknowledged.

\section{REFERENCES}

/1/SINGER,S.J. and NICHOLSON,G.L., Science 175(1972)720

/2/LUZZATI,V. in "Biological Membranes" (D.Chapman, ed.) vol.1, pp.71-123, Academic Press, London 1968

/3/SEDDON,J.M., Biochim.Biophys.Acta 1031(1990)1

/4/TATE,M.W.,EICKENBERRY,E.F.,TURNER,D.C.,SHYAMSUNDER, E. and GRUNER,S.M., Chem.Phys.Lipids $57(1991) 147$

/5/LINDBLOM,G. and RILFORS, L., Biochim. Biophys. Acta 998(1989)221

/6/KINNUNEN,P.K.J., Chem.Phys.Lipids 57(1991)375

/7/LAGGNER, P. in "New Methods in X-Ray Absorption, Scattering and Diffraction for Applications in Structural Biology" (B.Chance and H.D.Bartunik, eds) pp.171-182, Academic Press, London 1986

/8/LAGGNER,P., Top.Curr.Chem. 145(1988)145

/9/CAFFREY,M., Annu.Rev.Biophys.Biophys.Chem. 18(1989)159

/10/CAFFREY,M., Top.Cur.Chem.151(1989)75

/11/MILLS,D.M. in "Handbook on Synchrotron Radiation (G.Brown and D.E.Moncton, eds) vol.3, pp.291-335, North-Holland, Amsterdam 1991

/12/KOCH,M.H.J. in "Handbook on Synchrotron Radiation" (S.Ebashi, M.Koch and E.Rubenstein, eds) vol.4, pp. 241-268, North-Holland, Amsterdam 1991

/13/CAFFREY,M., MAGIN,R.L., HUMMEL,B. and ZHANG,J., Biophys.J. 58(1990)21

/14/RAPP,G. and GOODY,R.S., J.Appl.Cryst. 24(1991)836

/15/KRIECHBAUM,M., LAGGNER,P. and RAPP,G., Nucl.Instr.Meth.Phys.Res. A291(1990)41

/16/LAGGNER,P.and MIO,H., Nucl.Instr.Meth.Phys.Res. A323(1992)86

/17/ $\mathrm{KOCH}_{3}$ M.H.J., Proc. 4th Intern.Conf.on Biophysics and Synchrotron Radiation, Tsukuba, 1992, to be published by Oxford University Press, Oxford, UK

18/KRIECHBAUM,M., RAPP,G., HENDRIX,J. and LAGGNER,P., Rev.Sci.Instr. 60(1989)2541

/19/CHRISTIAN,J.W. in "Physical Metallurgy" (R.W.Kahn, ed.) pp.471-587, North-Holland 1970

/20/NISHIYAMA, Z. "Martensitic Transformation", Material Science Series

(M.Fine, M.Meshii and C.Wayman, eds) Academic Press, New York 1978

/21/JANIAK,M.J., SMALL,D.M. and SHIPLEY,G.G., Biochemistry 15(1976) 4575

/22/LAGGNER,P., KRIECHBAUM,M., RAPP,G. and HENDRIX,J., Proc. 2nd Europ. Conf. on Progress in X-Ray Synchrotron Radiation Research, Rome, 1989 (A.Balerna, E.Bernieri and S.Mobilio, eds) pp.995-998, Ital.Phys.Soc. Bologna 1990 